Corresponding authors: adubuc@bwh.harvard.edu; dhhwang@bwh.harvard.edu

(c) 2021 Terraf et al. This article is distributed under the terms of the Creative Commons Attribution-NonCommercial License, which permits reuse and redistribution, except for commercial purposes, provided that the original author and source are credited.

Ontology terms: chronic lymphatic leukemia; hematological neoplasm; lung adenocarcinoma

Published by Cold Spring Harbor Laboratory Press

doi:10.1101/mcs.a006089

\section{Twists and turns from "tumor in tumor" profiling: surveillance of chronic lymphocytic leukemia (CLL) leads to detection of a lung adenocarcinoma, whose genomic characterization alters the original hematologic diagnosis}

\author{
Panieh Terraf, ${ }^{1}$ Lynette M. Sholl, ${ }^{2}$ Matthew S. Davids, ${ }^{3}$ Mark M. Awad, ${ }^{3}$ \\ Elizabeth P. Garcia, ${ }^{4}$ Laura E. MacConaill, ${ }^{2}$ Paola Dal Cin, ${ }^{2}$ Annette Kim, ${ }^{2}$ \\ Neal I. Lindeman, ${ }^{2}$ Matthew Stachler, ${ }^{5}$ David H. Hwang, ${ }^{2,6}$ and Adrian M. Dubuc ${ }^{2,6}$ \\ ${ }^{1}$ Department of Pathology, Memorial Sloan Kettering Cancer Center, New York, New York 10065, USA; \\ ${ }^{2}$ Department of Pathology, Brigham and Women's Hospital and Harvard Medical School, Boston, \\ Massachusetts 02115, USA; ${ }^{3}$ Department of Medical Oncology, Dana-Farber Cancer Institute, Boston, \\ Massachusetts 02215, USA; ${ }^{4}$ Center for Advanced Molecular Diagnostics, Brigham and Women's Hospital, \\ Harvard Medical School, Boston, Massachusetts 02115, USA; ${ }^{5}$ Department of Pathology, University of \\ California San Francisco, San Francisco, California 94143, USA
}

Abstract Comprehensive characterization of somatic genomic alterations has led to fundamental shifts in our understanding of tumor biology. In clinical practice, these studies can lead to modifications of diagnosis and/or specific treatment implications, fulfilling the promise of personalized medicine. Herein, we describe a 78-yr-old woman under surveillance for long-standing untreated chronic lymphocytic leukemia (CLL). Molecular studies from a peripheral blood specimen revealed a TP53 p.V157F mutation, whereas karyotype and fluorescence in situ hybridization (FISH) identified a 17p deletion, trisomy 12, and no evidence of IGH-CCND1 rearrangement. Positron emission tomography-computed tomography scan identified multistation intra-abdominal lymphadenopathy and a pulmonary nodule, and subsequent pulmonary wedge resection confirmed the presence of a concurrent lung adenocarcinoma. Targeted next-generation sequencing of the lung tumor identified an EGFR in-frame exon 19 deletion, two TP53 mutations (p.P152Q, p.V157F), and, unexpectedly, a IGH-CCND1 rearrangement. Follow-up immunohistochemistry (IHC) studies demonstrated a cyclin D1-positive lymphoid aggregate within the lung adenocarcinoma. The presence of the TP53 p.V157F mutation in the lung resection, detection of an IGH-CCND1 rearrangement, and cyclin D1 positivity by IHC led to revision of the patient's hematologic diagnosis and confirmed the extranodal presence of mantle cell lymphoma within the lung mass, thus representing a "tumor in tumor." Manual review of the sequencing data suggested the IGH-CCND1 rearrangement occurred via an insertional event, whose size precluded detection by original FISH studies. Thus, routine imaging for this patient's known hematologic malignancy led to detection of an unexpected solid tumor, whose subsequent precision medicine studies in the solid tumor redefined the original hematological diagnosis.

[Supplemental material is available for this article.]

${ }^{6}$ These authors contributed equally to this work. 


\section{INTRODUCTION}

Advances in sequence-based technologies, coupled with large cohort studies, have revolutionized our understanding of cancer (Berger and Mardis 2018). Through comprehensive "omic" approaches, our knowledge of driver alterations has significantly expanded, facilitating the molecular dissection of morphologically similar entities (Papaemmanuil et al. 2016) and fueling drug discovery with response to specific genomic biomarkers (Wheler et al. 2016; Hillier et al. 2019; Nardi et al. 2020). Today, genomic profiling has become an important, and often integral, component of clinical care, providing critical diagnostic and prognostic information complementing, and at times superseding, histomorphological analysis alone (Frampton et al. 2013; Chau et al. 2016; Louis et al. 2016; Papaemmanuil et al. 2016).

Over the past 9 years, comprehensive genomic profiling has been offered for all patients with a cancer diagnosis seen at Brigham and Women's Hospital and the Dana-Farber Cancer Institute (Sholl et al. 2016), including detection of single-nucleotide variants, copy number aberrations, and structural rearrangements across 447 genes (Garcia et al. 2017). More than 30,000 cases have been profiled to date, revealing actionable genomic alterations and improving our understanding of the genomic landscape of many tumor types (Mandelker et al. 2015; Harris et al. 2016; Bi et al. 2017; Ramkissoon et al. 2017).

Herein, we describe the importance and unexpected benefits of genomic profiling in a patient with a reported history of chronic lymphocytic leukemia (CLL), whose surveillance led to the detection of a lung adenocarcinoma. Subsequently genomic studies of the lung cancer uncovered an unanticipated genomic finding that led to revision of the original diagnosis of the patient's hematologic malignancy.

\section{RESULTS}

\section{Clinical Presentation}

A 78-yr-old women presented to our institute with an 8-yr history of Rai Stage II CLL diagnosed at an outside institution and an absolute lymphocyte count of $5.4 \times 10^{10} \mathrm{~L}$. Flow cytometry on a peripheral blood specimen confirmed monotypic B-cell population of CD5- and CD19-positive cells, which notably expressed bright CD20 and bright surface kappa light chain and lacked expression for CD23 (Fig. 1A). Fluorescence in situ hybridization (FISH) studies identified trisomy 12 in $77.5 \%$ of nuclei, loss of TP53 in 18\% of nuclei, and a normal (i.e., nonrearrangement) pattern with IGH and CCND1 probes (Fig. 1B). Conventional karyotype studies were concordant with FISH results, detecting a stemline clone with trisomy 12 as the sole aberration (Fig. 1C) and two related sideline clones exhibiting parallel evolution toward loss of TP53. Specifically, both subclones demonstrate unique structural rearrangements that each independently result in loss of the TP53 locus-one demonstrating isochromosome 17q and the other an unbalanced rearrangement between Chromosomes 17 and 18 (Fig. 1D). Other prognostic factors evaluated include TP53 sequencing, which identified a p.V157F variant, interpreted as an oncogenic alteration; mutated IGHV (IGHV 2-5; 7.97\% mutated); negative ZAP-70 expression; and absence of a NOTCH1 c.7541_7542delCT mutation.

As part of routine surveillance, positron emission tomography-computed tomography (PET-CT) imaging was performed and unexpectedly identified a right upper lobe nodule, suspicious for a primary lung adenocarcinoma (Fig. 2A). A subsequent wedge resection and histopathology review confirmed a stage $1 \mathrm{~A}$ moderately differentiated adenocarcinoma (Fig. 2B). Further clinical review noted that the patient has never smoked by report. While this tumor was surgically resected, molecular profiling was performed as part of our institutional genomics program. 
A

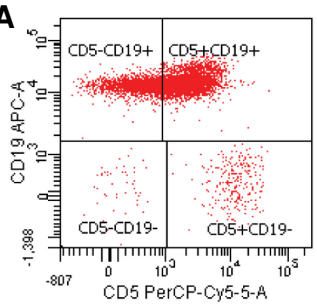

B

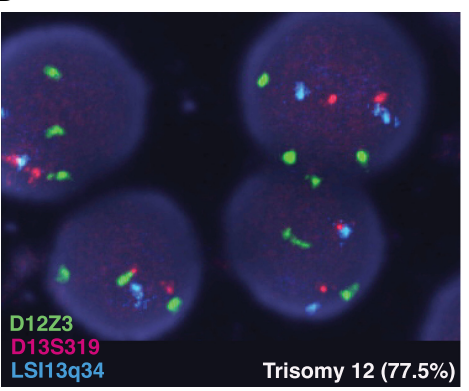

C

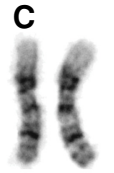

1

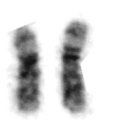

6

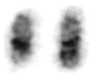

13

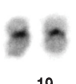

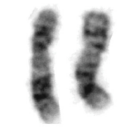

2
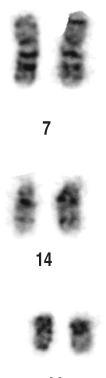
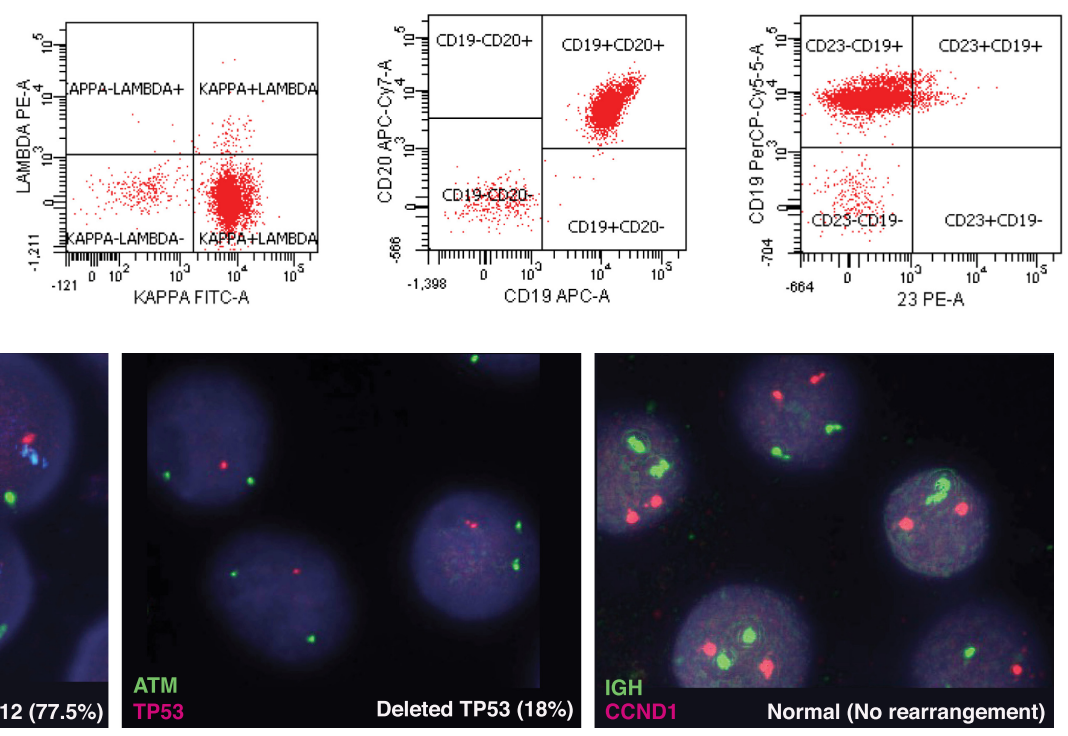

D

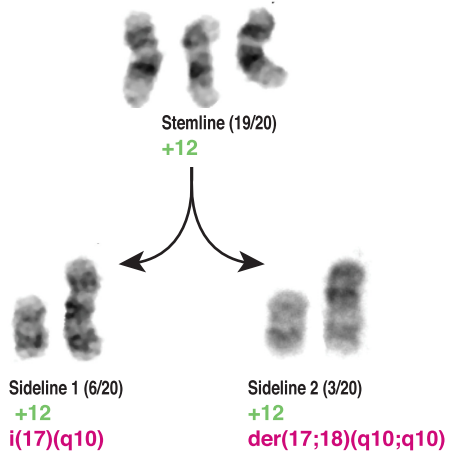

Figure 1. Immunophenotypic and cytogenetic characterization of a patient's known hematologic malignancy. (A) Flow cytometry identified an aberrant population of B-cell population positive for CD5 (dim), CD19, CD20 (bright), with monotypic kappa light chain. These cells were negative for CD23. (B) Interphase fluorescence in situ hybridization (FISH) studies using a chronic lymphocytic leukemia (CLL)-specific panel demonstrated trisomy 12 , detected in $77.5 \%$ of nuclei and loss of TP53 in $18 \%$ of nuclei, with no evidence of IGH-CCND1 rearrangement. (C) Chromosome studies identified a stemline clone with trisomy 12. (D) In addition to the stemline clone, chromosome studies identified two distinct sideline clones with differing structural variants resulting in loss of $17 p$.

\section{Comprehensive Genomic Profiling}

Molecular profiling of the lung tumor biopsy yielded an in-frame EGFR exon 19 deletion, whose presence is associated with responsiveness to EGFR tyrosine kinase inhibitors (Paez et al. 2004; Sullivan and Planchard 2016). In addition there were two TP53 missense variants (c.455C > A, p.P152Q, and c.469G > T, p.V157F), one of which was previously identified in patient's hematological malignancy. The remaining alterations were interpreted as variants of unknown clinical significance and presumed to reflect rare germline alterations (Table 1). 
A

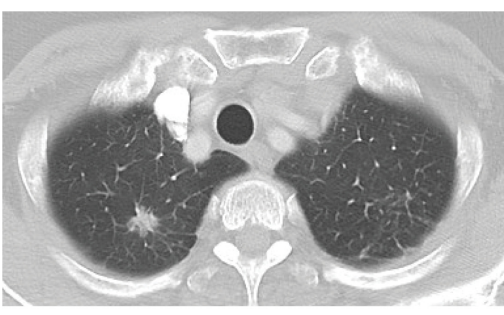

C

TP53 -c.469G>T(p.V157F) - 8\% c. $455 c>A(p . P 152 Q)-14 \%$;
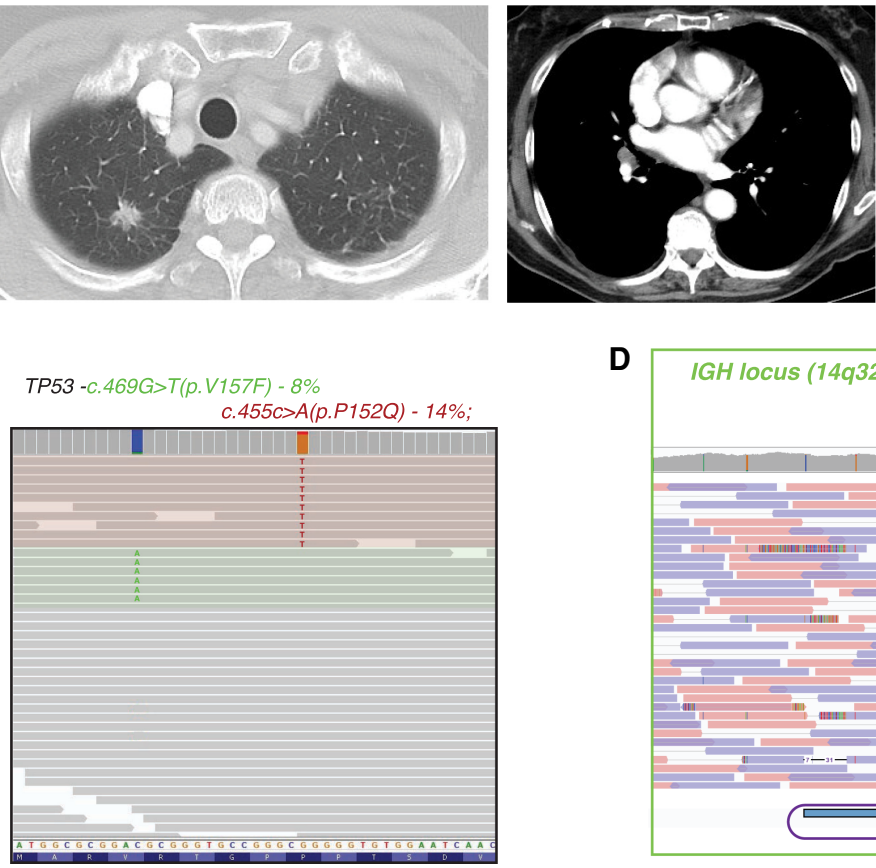

D
B

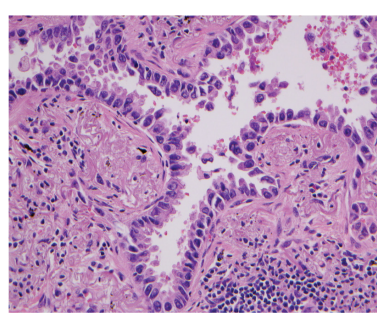

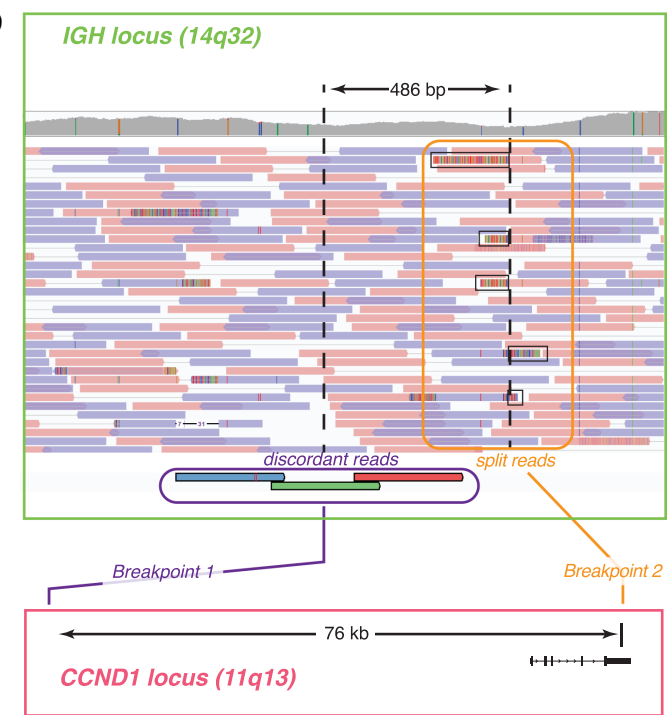

$\mathbf{F}$

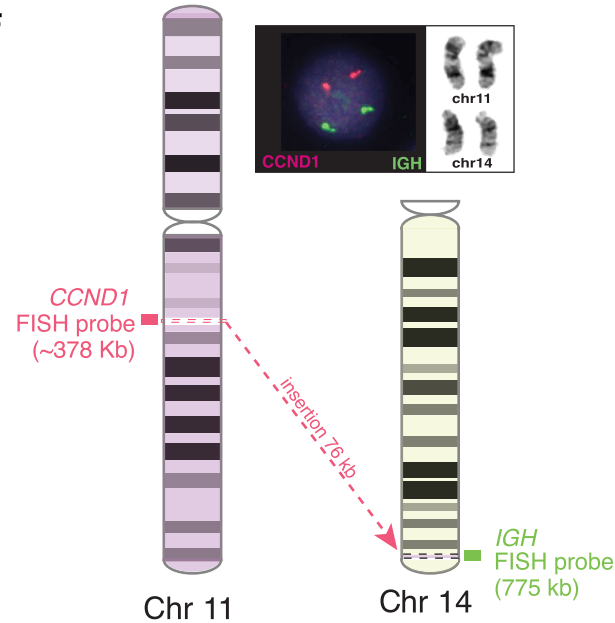

Chr 14
E
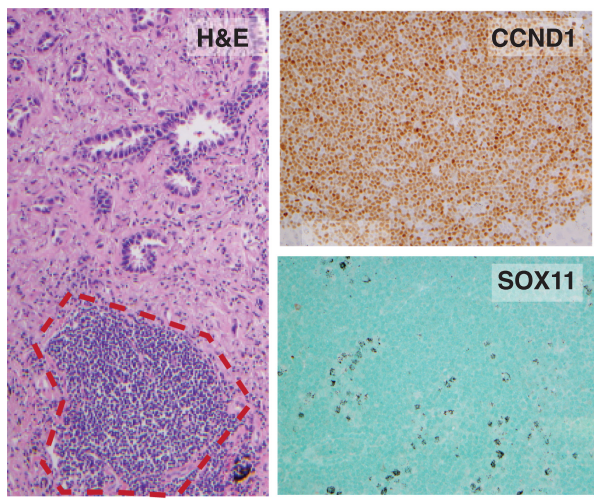

Figure 2. Detection and genomic characterization of patient's lung adenocarcinoma. (A) Positron emission tomography-computed tomography (PET-CT) imaging reveals an upper lobe node. (B) Hematoxylin and eosin staining of the resected biopsy confirms a lung adenocarcinoma. (C) Integrative Genomics Viewer (IGV) view of TP53 missense variants confirms alterations occurring in trans. (D) Schematic representation of the IGH locus and the detection of both split and discordant reads in IGV that map to the CCND1 locus. (E) (Left) Detection of focal lymphoid aggregate (within dotted area) in the lung adenocarcinoma; (upper right) CCND1 immunohistochemistry on lymphoid aggregates confirms expression; (lower right) absence of SOX11 expression in lymphoid aggregate. (F) Schematic demonstrating the insertion of CCND1 (11q13.3) into the IGH locus (14q32). The size of the FISH probes and false-negative cytogenetic studies are also shown. 


\begin{tabular}{|c|c|c|c|c|c|c|}
\hline Gene & Chromosome & HGVS DNA reference & $\begin{array}{l}\text { HGVS protein } \\
\text { reference }\end{array}$ & $\begin{array}{l}\text { Variant allele } \\
\text { fraction (\%) }\end{array}$ & $\begin{array}{l}\text { Sequencing } \\
\text { coverage }\end{array}$ & $\begin{array}{c}\text { Clinical } \\
\text { tier }\end{array}$ \\
\hline EGFR & 7 & c.2234_2249AGGAATTAAGAGAAGC > A & p.ELREA746del & 8 & 253 & 1 \\
\hline TP53 & 17 & c. $455 \mathrm{C}>\mathrm{A}$ & p.P152Q & 14 & 99 & 3 \\
\hline TP53 & 17 & c. $469 \mathrm{G}>\mathrm{T}$ & p.V157F & 8 & 105 & 3 \\
\hline DMD & $x$ & c. $8180 A>C$ & p.D2727A & 52 & 229 & 4 \\
\hline KMT2D & 12 & c. $15080 G>A$ & p.R5027Q) & 46 & 113 & 4 \\
\hline PRF1 & 10 & c. $658 \mathrm{G}>\mathrm{A}$ & p.G220S & 46 & 44 & 4 \\
\hline WRN & 8 & c. $2437 \mathrm{G}>\mathrm{T}$ & p.D813Y & 56 & 261 & 4 \\
\hline
\end{tabular}

Manual review of the TP53 variants demonstrated that both variants were on distinct alleles (Fig. 2C). Although the copy number landscape was largely unremarkable (Supplemental Fig. 1A), structural variant analysis identified a rearrangement between the $3^{\prime}$ untranslated region of CCND1 and the IGH locus. Given the unexpected nature of this finding, manual review of sequencing reads within the IGH locus was performed and identified a second breakpoint, based on discordant reads, mapping to the major translocation cluster region of CCND1, $76 \mathrm{~kb}$ upstream of the coding sequence (Fig. 2D). The presence of two distinct breakpoints within the IGH locus is most suggestive of a genomic insertional event that placed $76 \mathrm{~kb}$ of CCND1 and its $3^{\prime}$ UTR within the IGH enhancer region. Breakpoints were confirmed by reverse transcription polymerase chain reaction (RT-PCR) (Supplemental Fig. 1B,C).

\section{Reassessment of Initial Hematologic Malignancy}

Given the identification of a putative IGH-CCND1 rearrangement, immunohistochemical (IHC) studies were performed and confirmed cyclin D1 expression in lymphoid aggregates in the lung (Fig. 2E). Taken together with the TP53 p.V157F mutation previously identified in the context of the patient's reported CLL, these findings are most consistent with the diagnosis of extranodal involvement of the lung by mantle cell lymphoma (MCL), incidentally sampled with the adenocarcinoma. The MCL was subsequently shown to be negative for SOX11 expression (Fig. 2E). Given the importance of CCND1 status in the appropriate diagnosis of certain B-cell neoplasms, the discrepancy between the originally reported FISH studies (e.g., absence of IGH-CCND1 rearrangement) and molecular findings was further investigated. As the CCND1 FISH probe is $378 \mathrm{~kb}$, the insertion of CCND1 into the IGH locus resulted in displacement of only $20 \%$ (76/378) of the targeted genomic sequence. FISH studies were repeated, with knowledge of the underlying genomic event, but no appreciable differences in the relative intensity of CCND1 were observed. Thus, this insertion is not reliably detectable by FISH nor could this finding be detected by conventional karyotyping (Fig. 2F). The patient remained on observation for her hematologic malignancy, with regular visits three to four times per year that include history and physical exam and laboratory evaluation, as well as CT scans once or twice per year, which have not revealed significant progression over the course of the past 5 years.

\section{DISCUSSION}

Comprehensive genomic characterization can be essential to the diagnosis and appropriate clinical care of patients with cancer (Polonis et al. 2020). CLL and MCL are distinct and well- 
defined mature B-cell neoplasms, which, despite some overlap in their immunophenotype and genomics, are typically associated with drastically contrasting clinical course (Puente et al. 2018). Despite significant clinical heterogeneity in both entities, CLL is typically associated with an indolent disease course, whereas MCL is generally significantly more aggressive and carries a poor prognosis (Puente et al. 2015). Morphological assessment of nodal involvement is readily able to establish diagnosis in part through the use of $\mathrm{IHC}$ evaluation of CCND1 expression; however, in case of leukemic nonnodal presentation, establishing a precise diagnosis of these diseases requires the use of immunophenotypic and cytogenetic characteristics to establish diagnosis (Nelson et al. 2002, 2007; Fateh 2017). Although both entities are classically CD5-positive, CLL typically coexpresses CD23 as well, while expressing unusually dim CD20 and surface light chain. Although other markers, such as CD200, may also aid in the discrimination of these entities, these markers may not be widely available and immunophenotypic characteristics are not universally true. Accordingly, flow cytometry studies are frequently supplemented by cytogenetic analysis, relying on the detection or exclusion of IGH-CCND1 rearrangement, present in 95\% of MCLs (Mozos et al. 2009). Recently, two clinicopathologically distinct subgroups of MCL have been identified, largely differentiating conventional, nodal MCL (cMCL) from its nonnodal (leukemic) counterpart (nnMCL) (Clot et al. 2018). CMCL is prominently characterized by unmutated IGHV, SOX11 positivity, and frequent chromosomal aberrations accounting for $\sim 90 \%$ of cases (Navarro et al. 2020). In contrast, nnMCL demonstrate fewer chromosomal aberrations, a mutated IGHV status, SOX11 negativity, and, most importantly, a significantly improved overall survival compared to cMCL (Clot et al. 2018). Thus, differentiating $C L L$ from $M C L$, but also identifying the specific $M C L$ subgroup, is of critical clinical importance.

Herein, we describe a rare case of nnMCL with a cytogenetically cryptic IGH-CCND1 rearrangement. Immunophenotypic and cytogenetic studies were performed upon presentation to our institute. Although flow cytometry was more suggestive of $\mathrm{MCL}$, the findings did not definitely exclude the prior outside diagnosis of CLL. Furthermore, the cytogenetic studies (both FISH and karyotype) failed to identify an IGH-CCND1 rearrangement, and the aberrations that were detected-trisomy 12 and loss of TP53-although highly recurrent in $\mathrm{CLL}$, can also be found in MCL. Accordingly, the outside diagnosis remained in place. However, routine surveillance identified an upper lobe nodule, subsequently confirmed to be lung adenocarcinoma, and was studied via our institutional sequencing program. Sequencing studies not only identified an actionable alteration EGFR exon19 deletion, whose presence is associated with response to EGFR tyrosine kinase inhibitors, but also detected an unexpected IGH-CCND1 rearrangement. Subsequent histopathology review confirmed the presence of a focal lymphoid aggregate within the sequenced region of the lung adenocarcinoma, which was found to be positive for cyclin D1 expression by IHC. Thus we, unexpectedly, performed "tumor in tumor" sequencing. Further analysis of the sequencing data demonstrated that the rearrangement occurs through a genomic insertion event of the CCND1 gene into the IGH locus, a finding that is cytogenetically cryptic by FISH or karyotype, because of its size. Insertional events are rare, but recurrent findings are occasionally observed in several hematological malignancies, including MCL (Polonis et al. 2020). As sequence-based approaches with the capacity to detect rearrangement-and specifically enhancer hijackers-continue to be developed, a more accurate assessment of the frequency of these events may be possible.

This case highlights the importance and utility of genomic profiling in the context of clinical care. In the present case, the genomic studies performed demonstrated improved resolution over traditional diagnostic assays. Although the primary objective of sequencing was to characterize the patient's lung adenocarcinoma, these studies significantly, although unexpectedly, also improved our understanding of the patient's concurrent hematologic 
malignancy, which may take on even greater importance with regard to therapy selection once treatment is indicated. Discussion of atypical cases within an interdisciplinary team are particularly helpful to identify clinical scenarios in which additional or expanded genomic studies are necessary to sufficiently support diagnoses and management.

\section{METHODS}

\section{Cytogenetics}

FISH and conventional karyotyping were performed on a CpG-stimulated peripheral blood specimen. FISH studies included analysis of 200 interphase nuclei using the Abbott Vysis CLL FISH probe kit, whereas conventional karyotyping was reported on 20 metaphases, as previously described (Dubuc et al. 2016).

\section{Targeted Next-Generation Sequencing}

Targeted massively parallel sequencing was performed on DNA extracted from paraffin-embedded material. Exonic sequences from 300 genes of interest, as well as 113 introns (from 35 genes), were captured using a solution-phase Agilent SureSelect hybrid capture kit and sequenced on a Illumina HiSeq 2500 sequencer as previously described (Garcia et al. 2017). A total of 7,251,091 high-quality reads were aligned for this specimen, with a mean of 159 reads for all targeted exons, $96 \%$ of which demonstrate greater than 30 reads. Structural variant analysis was performed using the BreakMer algorithm (Abo et al. 2015).

\section{ADDITIONAL INFORMATION}

\section{Data Deposition and Access}

Variants have been deposited as part of AACR Project GENIE (https://genie.cbioportal.org/ patient?studyld=genie_public\&caseld=GENIE-DFCI-007672).

Competing Interest Statement

The authors have declared no competing interest.

Referees

Adam C. Smith

Anonymous

Received February 22, 2021; accepted in revised form May 3, 2021.

\section{Ethics Statement}

The patient provided written, informed consent (protocol 11-104). This study was approved by the institutional review board of the Dana-Farber Cancer Institute and a Mass General Brigham IRB.

\section{Author Contributions}

P.T., D.H.H., and A.M.D. acquired clinical data and images, performed the literature search, and wrote the report. M.S.D. and M.M.A. are responsible for the clinical management of the patient. M.S., D.H.H., and A.M.D. interpreted the molecular findings. All authors contributed to critical revision and final approval of the report.

\section{REFERENCES}

Abo RP, Ducar M, Garcia EP, Thorner AR, Rojas-Rudilla V, Lin L, Sholl LM, Hahn WC, Meyerson M, Lindeman $\mathrm{NI}$, et al. 2015. BreaKmer: detection of structural variation in targeted massively parallel sequencing data using kmers. Nucleic Acids Res 43: 1-13. doi:10.1093/nar/gku1303

Berger MF, Mardis ER. 2018. The emerging clinical relevance of genomics in cancer medicine. Nat Rev Clin Oncol 15: 353-365. doi:10.1038/s41571-018-0002-6 
Bi WL, Horowitz P, Greenwald NF, Abedalthagafi M, Agarwalla PK, Gibson WJ, Mei Y, Schumacher SE, BenDavid U, Chevalier A, et al. 2017. Landscape of genomic alterations in pituitary adenomas. Clin Cancer Res 23: 1841-1851. doi:10.1158/1078-0432.CCR-16-0790

Chau NG, Li YY, Jo VY, Rabinowits G, Lorch JH, Tishler RB, Margalit DN, Schoenfeld JD, Annino DJ, Goguen LA, et al. 2016. Incorporation of next-generation sequencing into routine clinical care to direct treatment of head and neck squamous cell carcinoma. Clin Cancer Res 22: 2939-2949. doi:10.1158/1078-0432.CCR$15-2314$

Clot G, Jares P, Giné E, Navarro A, Royo C, Pinyol M, Martín-Garcia D, Demajo S, Espinet B, Salar A, et al. 2018. A gene signature that distinguishes conventional and leukemic nonnodal mantle cell lymphoma helps predict outcome. Blood 132: 413-422. doi:10.1182/blood-2018-03-838136

Dubuc AM, Davids MS, Pulluqi M, Pulluqi O, Hoang K, Hernandez-Sánchez JM, Schlich C, Hernández-Rivas JM, Brown JR, Dal Cin P. 2016. FISHing in the dark: how the combination of FISH and conventional karyotyping improves the diagnostic yield in CpG-stimulated chronic lymphocytic leukemia. Am J Hematol 91: 978-983. doi:10.1002/ajh.24452

Fateh N. 2017. Mantle cell lymphoma misdiagnosed as chronic lymphocytic leukemia: optimization of diagnostic approach. J Clin Exp Oncol 6: 13-15. doi:10.4172/2324-9110.1000200

Frampton GM, Fichtenholtz A, Otto GA, Wang K, Downing SR, He J, Schnall-Levin M, White J, Sanford EM, An $P$, et al. 2013. Development and validation of a clinical cancer genomic profiling test based on massively parallel DNA sequencing. Nat Biotechnol 31: 1023-1031. doi:10.1038/nbt.2696

Garcia EP, Minkovsky A, Jia Y, Ducar MD, Shivdasani P, Gong X, Ligon AH, Sholl LM, Kuo FC, MacConaill $L E$, et al. 2017. Validation of OncoPanel, a targeted next-generation sequencing assay for the detection of somatic variants in cancer. Arch Pathol Lab Med 141: 751-758. doi:10.5858/arpa.20160527-OA

Harris $\mathrm{MH}$, DuBois SG, Bender JLG, Kim A, Crompton BD, Parker E, Dumont IP, Hong AL, Guo D, Church A, et al. 2016. Multicenter feasibility study of tumor molecular profiling to inform therapeutic decisions in advanced pediatric solid tumors. JAMA Oncol 2: 608-615. doi:10.1001/jama oncol.2015.5689

Hillier K, Hughes A, Shamberger RC, Shusterman S, Perez-Atayde AR, Wassner AJ, lafrate AJ, Dubuc A, Janeway KA, Rothenberg SM, et al. 2019. A novel ALK fusion in pediatric medullary thyroid carcinoma. Thyroid 29: 1704-1707. doi:10.1089/thy.2019.0041

Louis DN, Perry A, Reifenberger G, von Deimling A, Figarella-Branger D, Cavenee WK, Ohgaki H, Wiestler OD, Kleihues P, Ellison DW. 2016. The 2016 World Health Organization classification of tumors of the central nervous system: a summary. Acta Neuropathol 131: 803-820. doi:10.1007/s00401-0161545-1

Mandelker D, Dal Cin P, Jacene HA, Armand P, Stone RM, Lindeman NI. 2015. Refractory myeloid sarcoma with a FIP1L1-PDGFRA rearrangement detected by clinical high throughput somatic sequencing. Exp Hematol Oncol 4: 1-5. doi:10.1186/s40164-015-0026-x

Mozos A, Royo C, Hartmann E, De Jong D, Baró C, Valera A, Fu K, Weisenburger DD, Delabie J, Chuang SS, et al. 2009. SOX11 expression is highly specific for mantle cell lymphoma and identifies the cyclin D1-negative subtype. Haematologica 94: 1555-1562. doi:10.3324/haematol.2009.010264

Nardi V, Ku N, Frigault MJ, Dubuc AM, Tsai HK, Amrein PC, Hobbs GS, Brunner AM, Narayan R, Burke $M E$, et al. 2020. Clinical response to larotrectinib in adult Philadelphia chromosome-like ALL with cryptic ETV6-NTRK3 rearrangement. Blood Adv 4: 106-111. doi:10.1182/bloodadvances .2019000769

Navarro A, Beà S, Jares P, Campo E. 2020. Molecular pathogenesis of mantle cell lymphoma. Hematol Oncol Clin North Am 34: 795-807. doi:10.1016/j.hoc.2020.05.002

Nelson BP, Variakojis D, Peterson LAC. 2002. Leukemic phase of B-cell lymphomas mimicking chronic lymphocytic leukemia and variants at presentation. Mod Pathol 15: 1111-1120. doi:10.1097/01.MP.0000031710 .32235 .24

Nelson BP, Gupta R, Dewald GW, Paternoster SF, Rosen ST, Peterson LC. 2007. Chronic lymphocytic leukemia FISH panel: impact on diagnosis. Am J Clin Pathol 128: 323-332. doi:10.1309/ 21TN2RUWKR827UW2

Paez JG, Jänne PA, Lee JC, Tracy S, Greulich H, Gabriel S, Herman P, Kaye FJ, Lindeman N, Boggon TJ, et al. 2004. EGFR mutations in lung, cancer: correlation with clinical response to gefitinib therapy. Science 304: 1497-1500. doi:10.1126/science.1099314

Papaemmanuil E, Gerstung M, Bullinger L, Gaidzik VI, Paschka P, Roberts ND, Potter NE, Heuser M, Thol F, Bolli $N$, et al. 2016. Genomic classification and prognosis in acute myeloid leukemia. N Engl J Med 374: 2209-2221. doi:10.1056/NEJMoa1516192

Polonis K, Schultz MJ, Olteanu H, Smadbeck JB, Johnson SH, Vasmatzis G, Xu X, Greipp PT, Ketterling $\mathrm{RP}$, Hoppman NL, et al. 2020. Detection of cryptic CCND1 rearrangements in mantle cell lymphoma by next generation sequencing. Ann Diagn Pathol 46: 151533. doi:10.1016/j.anndiagpath.2020.151533 
Puente XS, Beà S, Valdés-Mas R, Villamor N, Gutiérrez-Abril J, Martín-Subero Jl, Munar M, Rubio-Pérez C, Jares $P$, Aymerich $M$, et al. 2015. Non-coding recurrent mutations in chronic lymphocytic leukaemia. Nature 526: 519-524. doi:10.1038/nature14666

Puente XS, Jares P, Campo E. 2018. Chronic lymphocytic leukemia and mantle cell lymphoma: crossroads of genetic and microenvironment interactions. Blood 131: 2283-2296. doi:10.1182/blood-2017-10764373

Ramkissoon SH, Bandopadhayay P, Hwang J, Ramkissoon LA, Greenwald NF, Schumacher SE, O'Rourke R, Pinches N, Ho P, Malkin H, et al. 2017. Clinical targeted exome-based sequencing in combination with genome-wide copy number profiling: precision medicine analysis of 203 pediatric brain tumors. Neuro Oncol 19: 986-996. doi:10.1093/neuonc/now294

Sholl LM, Do K, Shivdasani P, Cerami E, Dubuc AM, Kuo FC, Garcia EP, Jia Y, Davineni P, Abo RP, et al. 2016. Institutional implementation of clinical tumor profiling on an unselected cancer population. JCI Insight 1: 1-19. doi:10.1172/jci.insight.87062

Sullivan I, Planchard D. 2016. Next-generation EGFR tyrosine kinase inhibitors for treating EGFR-mutant lung cancer beyond first line. Front Med 3: 1-13. doi:10.3389/fmed.2016.00076

Wheler JJ, Janku F, Naing A, Li Y, Stephen B, Zinner R, Subbiah V, Fu S, Karp D, Falchook GS, et al. 2016. Cancer therapy directed by comprehensive genomic profiling: a single center study. Cancer Res 76: 3690-3701. doi:10.1158/0008-5472.CAN-15-3043 


\section{COLD SPRING HARBOR Molecular Case Studies}

\section{Twists and turns from "tumor in tumor" profiling: surveillance of chronic lymphocytic leukemia (CLL) leads to detection of a lung adenocarcinoma, whose genomic characterization alters the original hematologic diagnosis}

Panieh Terraf, Lynette M. Sholl, Matthew S. Davids, et al.

Cold Spring Harb Mol Case Stud 2021, 7: a006089 originally published online June 1, 2021 Access the most recent version at doi: $10.1101 /$ mcs.a006089

Supplementary Material

References

License

Email Alerting Service
http://molecularcasestudies.cshlp.org/content/suppl/2021/06/21/mcs.a006089.D C1

This article cites 27 articles, 7 of which can be accessed free at: http://molecularcasestudies.cshlp.org/content/7/4/a006089.full.html\#ref-list-1

This article is distributed under the terms of the Creative Commons Attribution-NonCommercial License, which permits reuse and redistribution, except for commercial purposes, provided that the original author and source are credited.

Receive free email alerts when new articles cite this article - sign up in the box at the top right corner of the article or click here. 